\title{
LAND/GROOVE RECORDING SCHEME ON MSR MEDIA
}

\author{
M.Mieda, Y.Murakami, T.Inui, A.Takahashi and K.Ohta \\ Precision Technology Development Center, Sharp Corporation, \\ 2613-1, Ichinomoto-cho, Tenri, Nara 632, Japan
}

\begin{abstract}
Land/groove recording properties on a magnetically induced super-resolution (MSR) disk has been investigated. We prepared an exchange coupled type of MSR disk with double magnetic layers and examined the read/write characterisitics for various track pitches with magnetic field modulation recording. As the results, a track pitch of 0.65 $\mu \mathrm{m}$ was available for practical use from the point of view on crosstalk and crosswrite properties. We have also developed a new groove format in order to obtain address information on both lands and grooves.
\end{abstract}

KEYWORDS: MAGNETOOPTICAL, MAGNETICALLY INDUCED SUPER-RESOLUTION, LAND/GROOVE RECORDING, DISK FORMAT, GROOVE SHAPE.

\section{INTRODUCTION}

We have studied land/groove recording in a magnetically induced super-resolution (MSR) disk in order to realize larger capacity magneto-optical (MO) disks. Land/groove recording technology is one of effective methods, in which both land and groove areas are utilized as data area. In a conventional land or groove recording method, tracking error signal becomes smaller when track pitch becomes narrower. In addition, it is difficult to make narrow guide tracks precisely. On the other hand in land/groove recording, the widths of lands and grooves are set as the same, so larger tracking error signal can be detected and the groove forming process is not difficult.

In order to realize land/groove recording with higher track density, larger ability for crosstalk cancellation is required to recording media. So we employed an MSR medium. The MSR medium mainly consists of readout and recording magnetic layers, which are exchange-coupled with each other. It features that the magnetization direction of the readout layer is in-plane at room temperature and becomes perpendicular at high temperature. By the use of such a readout magnetic layer, we can obtain higher readout resolution without any external magnetic fields for reading out data [1,2]. The MSR disk has advantages of not only higher linear density but also higher track density. In the MSR disk, MO signal crosstalk from adjacent tracks is smaller than that in currently commercial-use MO disks, because the crosstalk signal is reduced by the presence of optical mask region in the focused readout beam spot. The optical mask region is formed by the in-plane magnetization of the readout layer with low temperature. We have already reported that a crosstalk of $-28 \mathrm{~dB}$ was obtained in land/groove recording with a $0.7 \mu \mathrm{m}$ track pitch in the MSR disk [3].
The MSR medium is preferable at the point of view on reducing crosstalk, but we have to solve a problem of how to obtain address information from both lands and grooves. In Mini-Disc (MD) and Compact-Disc-Recordable (CD-R), data are recorded only in grooves, and the grooves are wobbled to obtain address information. However, we can not detect address information on lands. In order to obtain address information from both lands and grooves, we developed one side wobbled grooves in which only one side wall of grooves was wobbled.

In this paper, we firstly describe land/groove recording characteristics in an MSR disk. We mainly report the crosstalk and crosswrite properties for various track pitches. The crosswrite property is one of important factors as well as the crosstalk in order to determine the lower limit of track pitch for practical use.

Next, we introduce a new disk format with one side wobbled grooves to obtain address information both on lands and grooves. The reproduced signal qualities for various shapes of wobbled grooves are reported and we indicate the preferable shape for practical use.

\section{LAND/GROOVE RECORDING IN MSR DISK}

\section{MSR disk structure and the readout resolution}

Figure 1 shows the exchange coupled type of MSR disk structure in this study. It features that an in-plane magnetization magnetic layer is used for a readout layer. Therefore it does not need readout magnetic field. The magnetization direction of the readout layer changed from in-plane to perpendicular at $150{ }^{\circ} \mathrm{C}$ in the prepared MSR disk. Curie temperatures of readout and recording layers were 360 and $220^{\circ} \mathrm{C}$, respectively. We used glass substrates, and grooves were directly formed by a reactive ion etching method [4]. In order to estimate land/groove recording characteristics, we set the 


\begin{tabular}{ll}
\hline \multicolumn{2}{c}{ Glass substrate } \\
\hline AIN interference layer & $65 \mathrm{~nm}$ \\
\hline GdFeCo readout layer & $50 \mathrm{~nm}$ \\
\hline DyFeCo recording layer & $50 \mathrm{~nm}$ \\
\hline AlN protective layer & $20 \mathrm{~nm}$ \\
\hline UV resin protective film & $10 \mu \mathrm{m}$ \\
\hline
\end{tabular}

Fig. 1. MSR disk structure in this study,

widths of lands and grooves as the same. In this paper, a track pitch of $0.7 \mu \mathrm{m}$ means that lands and grooves have the same width of $0.7 \mu \mathrm{m}$. The MSR medium had high recording magnetic field sensitivity, so we could overwrite data by magnetic field modulation (MFM) recording. MFM recording is suitable for mark edge recording, which can make the recording density about 1.5 times higher in comparison with conventional mark position recording. An external recording magnetic field was $\pm 15 \mathrm{kA} /$ $\mathrm{m}$ to obtain good overwriting performance in this study.

Figure 2 shows the readout resolution in the MSR disk compared with a conventional MO disk. The vertical axis indicates carrier-to-noise ratio (CNR) and the horizontal axis shows recorded mark length. The track pitch was $0.7 \mu \mathrm{m}$ and the groove depth was $40 \mathrm{~nm}$. An experimental optical head had a laser wavelength of $680 \mathrm{~nm}$ and a numerical aperture (N.A.) of 0.55 . The linear velocity was $5 \mathrm{~m} / \mathrm{s}$.

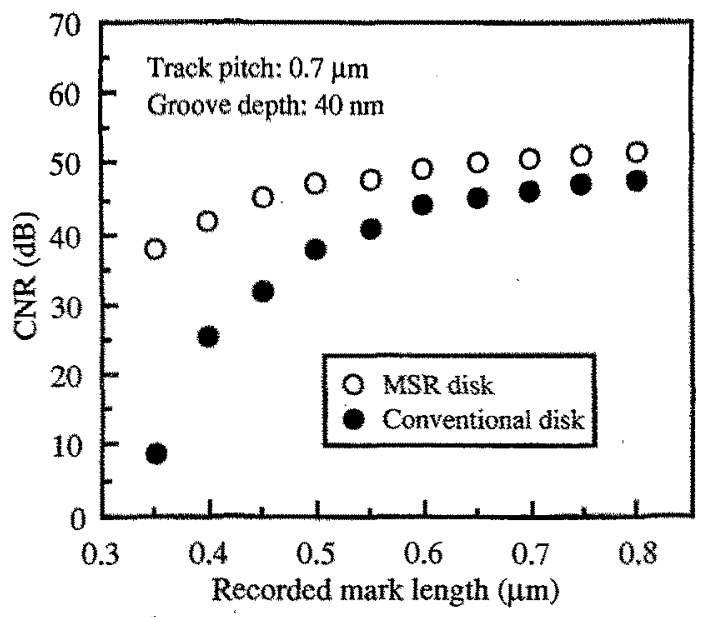

Fig. 2. CNR dependence on recorded mark length. A conventional disk consisted of glass substrate / AIN / DyFeCo/AIN/Al.
A conventional medium consisted of AIN, DyFeCo, $A I N$ and $A 1$ reflective layers, as well as currently commercial-use MO disks. The conventional disk had the same $0.7 \mu \mathrm{m}$-track pitch.

The CNR of the conventional disk is almost zero at a recorded mark length of $0.35 \mu \mathrm{m}$, which corresponds to the spatial cut-off frequency 2 N.A./ $\lambda$ of the optic system. On the other hand in the MSR disk, the recorded signal can be detected even under the cut-off frequency. At a recorded mark length of $0.45 \mu \mathrm{m}$, the CNR of more than $45 \mathrm{~dB}$ was obtained.

\section{Groove depth dependence of MO signal}

We prepared MSR disks with various groove depths and examined the CNR and crosstalk by a conventional MFM method. The crosstalk in this study means the value between adjacent lands and grooves, and it was defined as the ratio of the readout signal amplitude on a recorded track to that on an unrecorded track whose adjacent tracks were recorded. Figure 3 shows the result. The crosstalk decreased with increasing groove depth. On the other hand, the CNR also diminished with increasing groove depth because of the decrease of carrier level. The crosstalk value was $-32 \mathrm{~dB}$ even at the relatively shallow groove depth of $40 \mathrm{~nm}$, and it is low enough for practical use. From the point view on increasing linear density, the groove depth around $40 \mathrm{~nm}$ is preferable because the degradation of CNR is small.

\section{Track pitch dependence of MO signal}

In order to know the lower limit of track pitch sufficient for practical use, we examined the depen-

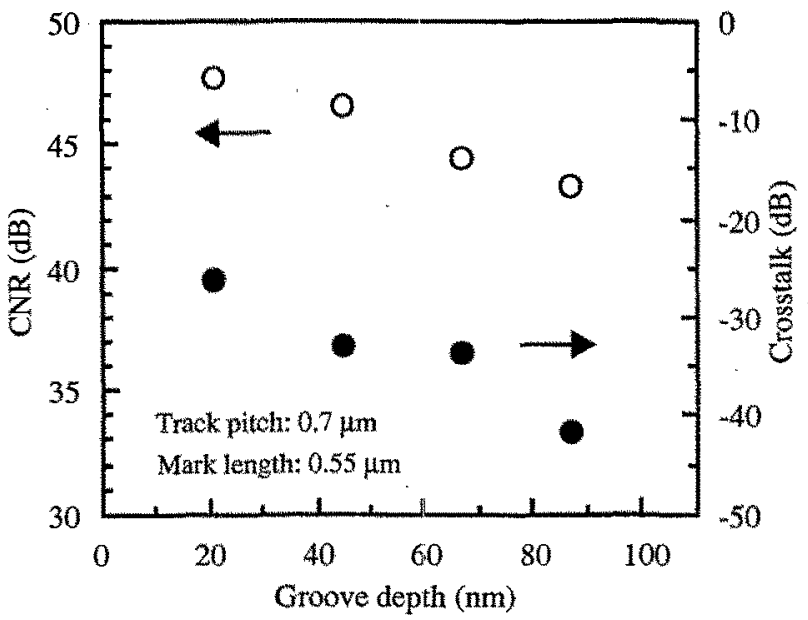

Fig. 3. CNR and crosstalk as functions of groove depth. 


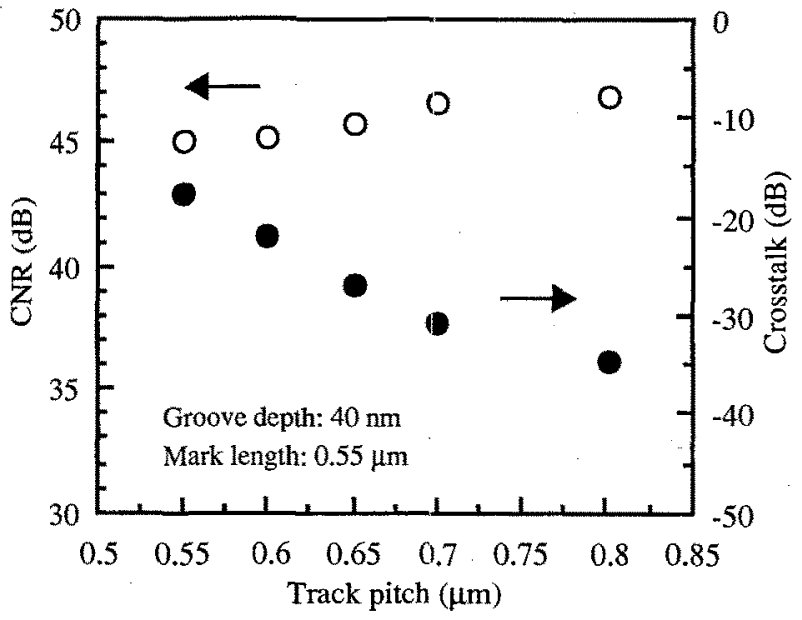

Fig. 4. CNR and crosstalk as functions of track pitch.

dence of MO signal quality on track pitch. The groove depth was $40 \mathrm{~nm}$ and the recorded mark length was $0.55 \mu \mathrm{m}$.

Figure 4 shows CNR and crosstalk dependence on track pitch. The crosstalk dependence on track pitch was much larger than that of CNR. The result shows that we can set the track pitch to $0.65 \mu \mathrm{m}$ from the point of view on crosstalk, where the crosstalk value was $-27 \mathrm{~dB}$. However if electrical crosstalk cancellation techniques are employed, the track pitch could be set narrower. It has already reported that crosstalk of about $-17 \mathrm{~dB}$ is permitted by the use of a PRML technique [5]. By the combination of our MSR disk and the PRML method, it will be possible to set the track pitch to $0.55 \mu \mathrm{m}$ from Fig. 4.

By the way, it is obvious that we have to consider crosswrite property to realize land/groove recording with narrow track pitch. We examined the property by MFM recording for track pitches of 0.6 , $0.65,0.7$ and $0.8 \mu \mathrm{m}$. The linear velocity was $5 \mathrm{~m} /$ $\mathrm{s}$.

Before estimating crosswrite property, we measured the recording power sensitivity for each track pitch. The recording laser power, where the CNR saturated, did not depend on track pitch and the value was around $5.5 \mathrm{~mW}$. So for all pitches, we wrote pre-recorded data with $5.6 \mathrm{~mW}$ to estimate crosswrite property. The pre-recorded mark length was $1 \mu \mathrm{m}$. After pre-recording on a land, we did an overwriting at the neighboring grooves. After that, the carrier level of pre-recorded signal was measured.

Figures 5 (a) and (b) show the results in crosswrite property and recording sensitivity for 0.8 $\mu \mathrm{m}$ and $0.6 \mu \mathrm{m}$-pitch, respectively. In the $0.8 \mu \mathrm{m}$ track pitch, degradation of the carrier level began at $7.8 \mathrm{~mW}$ and the degradation of $0.5 \mathrm{~dB}$ occurred at $8.0 \mathrm{~mW}$. On the other hand in the track pitch of $0.6 \mu \mathrm{m}$, shown in Fig. 5 (b), the degradation occurred at $5.4 \mathrm{~mW}$. The recording power margin was nearly zero at the track pitch of $0.6 \mu \mathrm{m}$

Figure 6 shows summary results in crosswrite for all pitches. Open circles denote the saturating laser power where CNR saturated, closed circles denote the disturbing laser power where $0.5 \mathrm{~dB}$ degradation of carrier level occurred. If the recording power margin of $6.0 \mathrm{~mW} \pm 10 \%$ is allowable in MFM type drives, we could set the track pitch to $0.65 \mu \mathrm{m}$ from the point of view on crosswrite. At

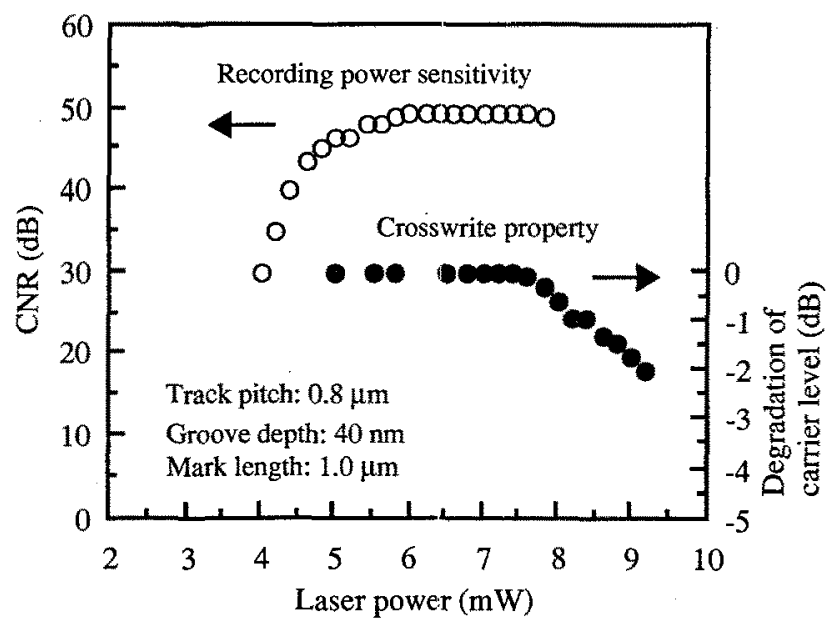

(a)

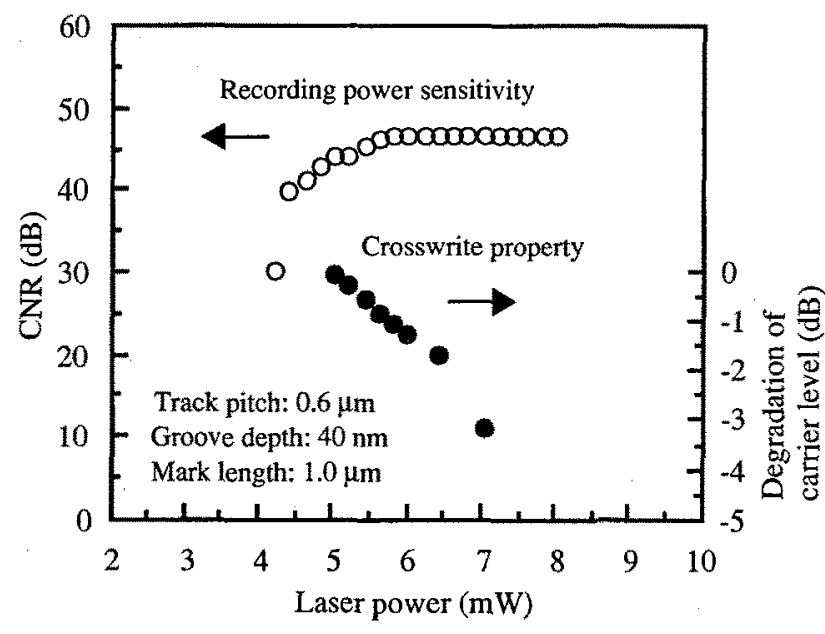

(b)

Fig. 5. Recording power sensitivity and crosswrite property: (a) at a track pitch of $0.8 \mu \mathrm{m}$, (b) at a track pitch of 0.6 $\mu \mathrm{m}$. 


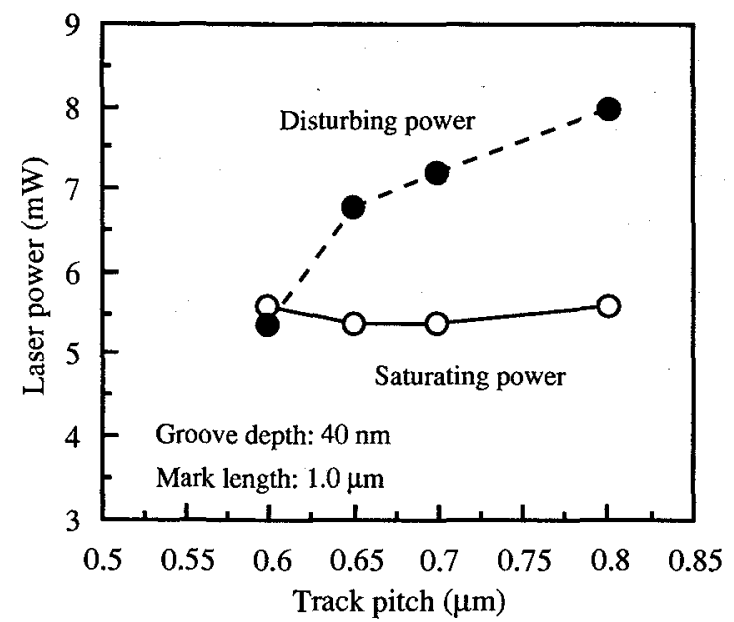

Fig. 6. The dependence of saturaring and disturbing laser power on track pitch. The saturating power means that the value where CNR saturated. The disturbing power means that the value where $0.5 \mathrm{~dB}$-degradation of carrier level occurred in crosswrite measurement.

this track pitch, crosstalk was about $-27 \mathrm{~dB}$, so as for crosstalk, any electric cancellation techniques will be unnecessary to realize land/groove recording on the MSR disk.

We consider that an MFM recording method with pulsed laser irradiation [6] is effective to improve crosswrite property. By combining the recording method with our MSR disk, narrower track pitch than $0.65 \mu \mathrm{m}$ will be realized in land/groove recording.

(a) from groove

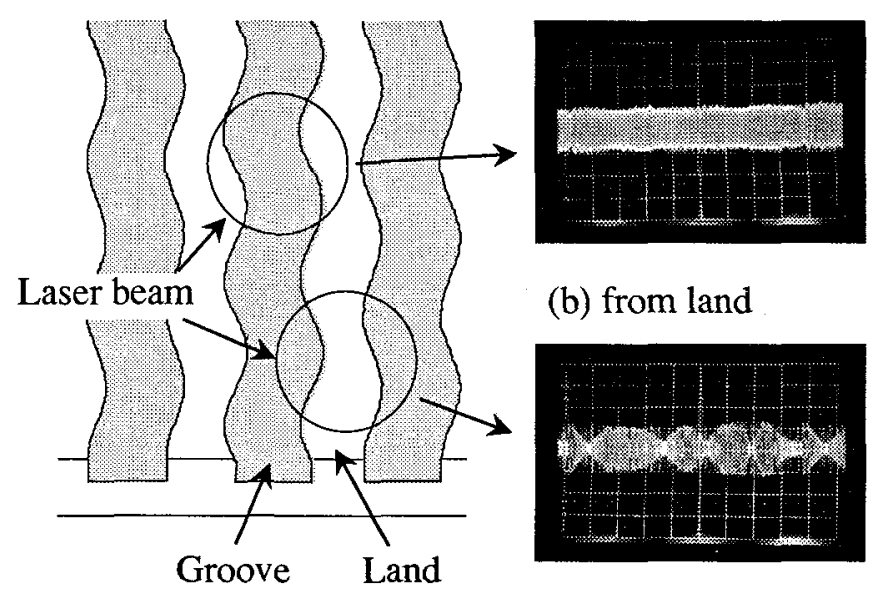

Fig. 7. Schematic view of conventional wobbled groove format and photos of the reproduced wobble signal: (a) from groove, (b) from land.

\section{ONE SIDE WOBBLED GROOVES SUITABLE FOR LAND/GROOVE RECORDING}

\section{Wobbled groove format}

Figure 7 illustrates a schematic view of conventional wobbled groove format. Currently MD and CD-R disks employ the groove recording method and the width of grooves is set wider than that of lands. Besides, grooves are periodically wobbled with a constant mean frequency. We can obtain address information by reproducing and demodulating the frequently wobbled grooves at the same time reading out recorded data. Photo (a) and (b) in Fig. 7 show reproduced wobble signal by the push-pull detection method from a groove and a land, respectively. As shown in Photo (b), the wobble signal was not be detected from a land because the two side walls of a land had different wobble signal.

In order to obtain favorable quality of wobble signal from both lands and grooves, we proposed one side wobbled groove format shown in Fig. 8. The groove shape features that only one side wall of grooves is wobbled. Therefore, we can get good quality of wobble signal from both lands and grooves, as observed on Photo (a) and (b) in Fig. 8. The one side wobbled grooves were formed by the use of a double beam type of cutting machine. Only one beam was wobbled and the other was kept straight, as shown in Fig. 9.

\section{Preferable shape for wobbled groove}

We prepared one side wobbled grooves with several shapes and estimated their reproduced sig-

(a) from groove
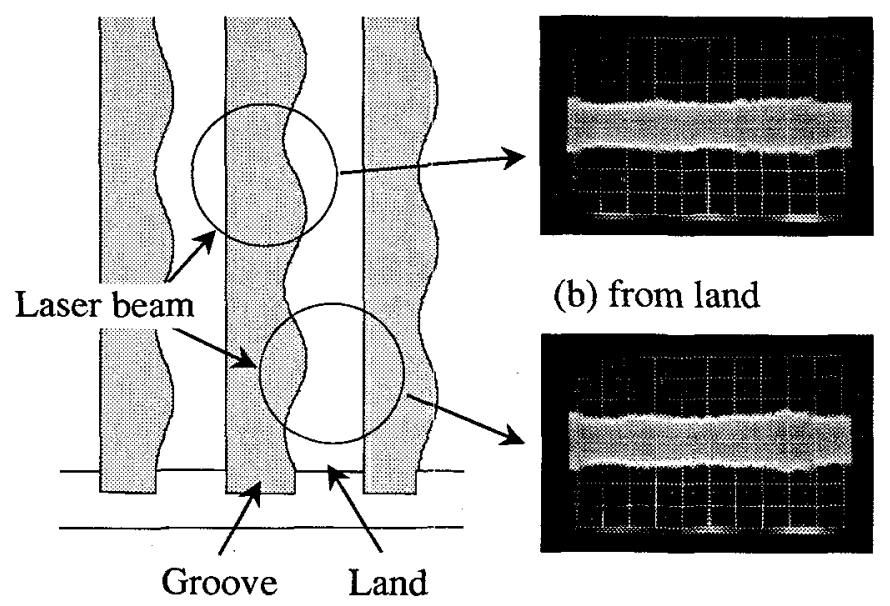

(b) from land

Fig. 8. Schematic view of proposed one side wobbled groove format and photos of the reproduced wobble signal: (a) from groove, (b) from land. 


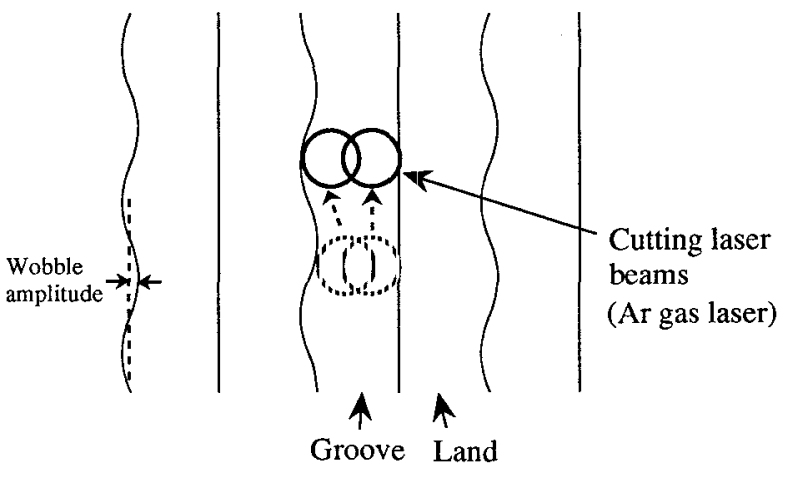

Fig. 9. Schematic diagram of forming method for one side wobbled grooves.

nal quality to know preferable shape for practical use. The mean frequency of the wobble signal was set about $80 \mathrm{kHz}$ at the linear velocity of $5 \mathrm{~m} / \mathrm{s}$.

The dependence of reproduced signal intensity on wobble amplitude is shown in Fig. 10. The value of wobble amplitude is shown in Fig. 9. The CNR of the wobble signal was detected from a tracking error signal, and the resolution bandwidth was set at $3 \mathrm{kHz}$. We set the reproduced laser power to 2.5 $\mathrm{mW}$ because that power is necessary to read out recorded information in the MSR disk. The medium reflectivity was about $25 \%$ in grooves.

Fig. 10 shows clearly that almost the same CNR values were obtained between a land and a groove. In our experimental detection circuit, a CNR of more than $35 \mathrm{~dB}$ was necessary to decode address information precisely. From this result, we think that

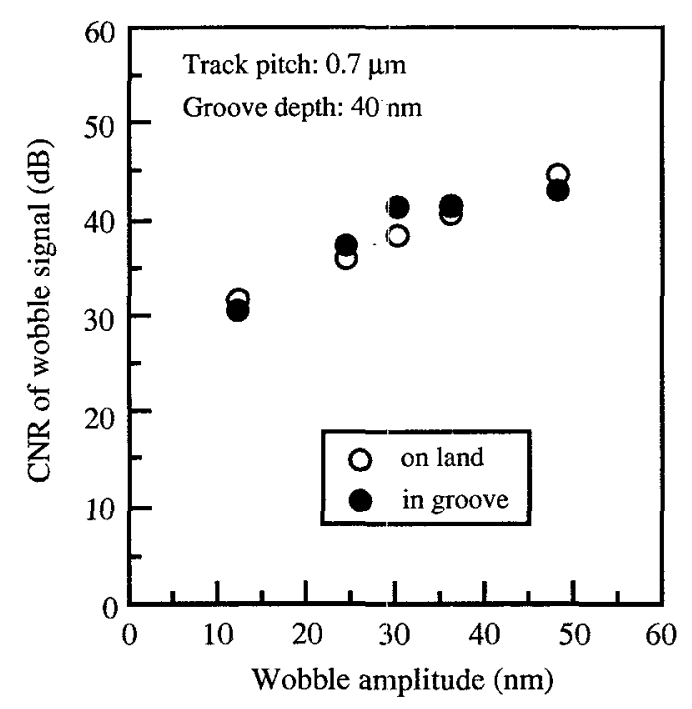

Fig. 10. CNR of reproduced wobble signal versus wobble amplitude.

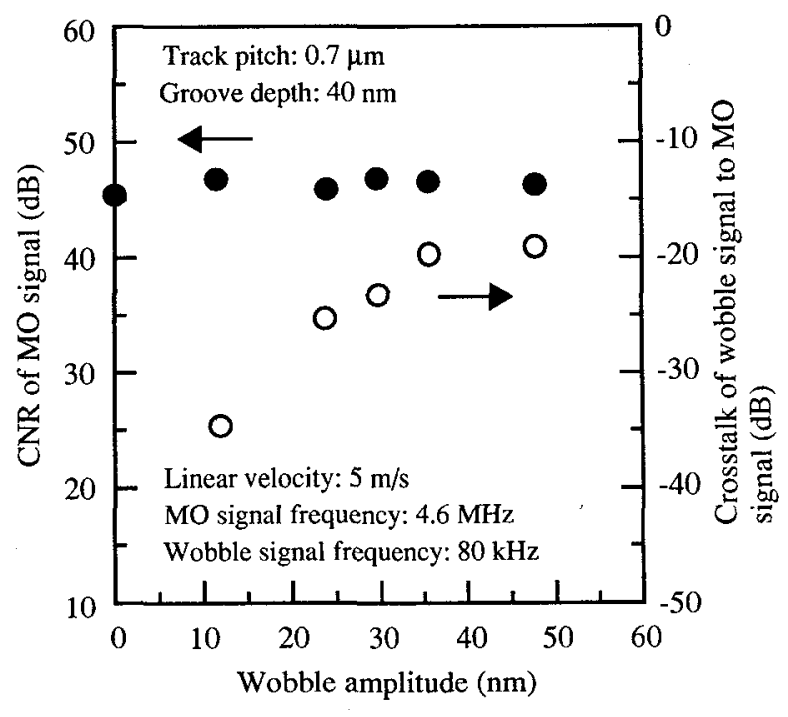

Fig. 11. CNR of MO signal and crosstalk of wobble signal to MO signal versus wobble amplitude.

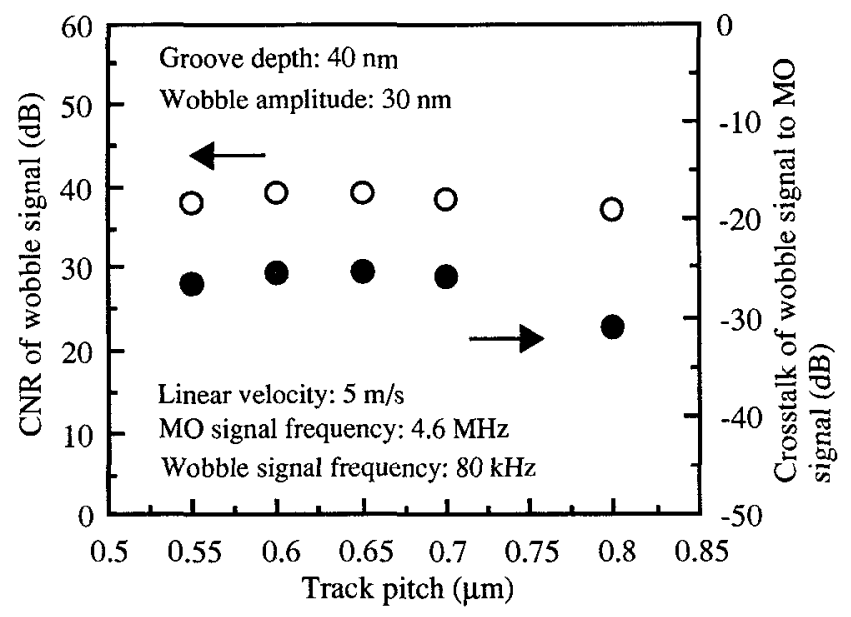

Fig. 12. CNR of wobble signal and crosstalk of wobble signal to MO signal versus track pitch.

wobble amplitude over $24 \mathrm{~nm}$ will be needed in practical use.

We also examined crosstalk of wobble signal to MO signal. If the crosstalk is too large, an electric frequency-filter will be needed to distinguish wobble signal and MO signal. Figure 11 shows the result. We defined the crosstalk as the difference of carrier levels between wobble signal and MO signal on an MO signal detection circuit. The crosstalk value of $-25 \mathrm{~dB}$ was low enough to get a bit error rate less than $1 \times 10^{-6}$ in our experiment with no electric filter that cuts off the frequency band of wobble signal. As the result, we selected the wobble amplitude of $30 \mathrm{~nm}$. Figure 11 also shows the CNR 
of MO signal as functions of wobble amplitude. The CNR values were almost the same in the range of the wobble amplitude from 0 to $50 \mathrm{~nm}$.

Next, we examined the wobble signal crosstalk to MO signal for various track pitches. Figure 12 shows the result. The variations of both CNR and crosstalk of wobble signal were little in the range of the track pitch from 0.55 to $0.8 \mu \mathrm{m}$. These results tell us that the one side wobbled groove method can be used even at the track pitch of $0.55 \mu \mathrm{m}$.

\section{CONCLUSIONS}

Land/groove recording characteristics for various track pitches were studied in an MSR disk by the use of a conventional MFM recording method. From the point of view on readout crosstalk and crosswrite properties, the track pitch of $0.65 \mu \mathrm{m}$ was available for practical use under a laser wavelength of $680 \mathrm{~nm}$ and an N.A. of 0.55 without any crosstalk cancellation techniques. We also developed a new disk format with one side wobbled grooves. Good signal quality of address information was obtained on both lands and grooves at a wobble amplitude of $30 \mathrm{~nm}$. The new format could be adapted to the $0.55 \mu \mathrm{m}$-track pitch, because the wobble signal crosstalk to MO signal was low enough for practical use.
In order to make the capacity larger in the MSR disk system, we consider that a pulsed laser irradiation type of MFM recording method and PRML signaling technique are quite effective. The former can reduce thermal interference during recording and result in improvement for crosswrite property. The latter technique can reduce readout crosstalk and also improve linear recording density. If we combine these techniques with the MSR disk in this study, it will be possible to reach an areal density of $3.5 \mathrm{Gbit} / \mathrm{in}^{2}$.

\section{REFERENCES}

[1] Y. Murakami, N. Iketani, J. Nakajima, A. Takahashi, K. Ohta and T. Ishikawa: J. Magn. Soc. Jpn. 17 (1993) Suppl. No.S1, p.201.

[2] J. Hirokane, Y. Murakami, H. Katayama, A. Takahashi, K. Ohta and H. Yamaoka: Proc. SPIE 2338 (1994) 301.

[3] Y. Murakami, A. Takahashi and S. Terashima: IEEE Trans. Magn. 31 (1995) NO.6, 3215.

[4] T. Inui, J. Hirokane, K. Ohta, H. Yamaoka and T.Ishikawa: Proc. SPIE 1078 (1989) 204.

[5] T. Iwanaga and M. Nakada: IEEE Trans. Magn. 31 (1995) NO.6, 3221.

[6] S. Yonezawa and M. Takahashi: Applied Optics, Vol.33, No.12 (1994) 2333. 